\title{
Effect of response effort requirement on relative frequency of short interresponse times: CRF and FR-5 reinforcement schedules
}

\author{
HARVARD L. ARMUS \\ University of Toledo, Toledo, Ohio
}

\begin{abstract}
Higher levels of required response effort in an operant task resulted in a greater relative frequency of short (less than $1 \mathrm{sec}$ ) interresponse times for both continuous reinforcement and fixedratio 5 (FR-5) groups, with the effect being accentuated for the FR-5 condition.
\end{abstract}

In 1986, Armus reported that the relative frequency of short (less than $1 \mathrm{sec}$ ) interresponse times in an operant task was an increasing function of the effort required to operate the lever. The greater the required lever operating force, the larger the ratio of presses with interresponse times (IRTs) of less than $1 \mathrm{sec}$ to total number of presses. The absolute number of short IRTs was, however, quite small. In the present study, a fixed-ratio 5 (FR-5) reinforcement schedule was introduced in an attempt to increase the number of short IRT responses relative to the total number of responses. In addition, the effect of response effort on the relative frequency of short IRTs was assessed for both continuous reinforcement (CRF) and FR-5 reinforcement schedules.

\section{METHOD}

\section{Subjects and Apparatus}

The subjects were 19 male Long-Evans strain rats, approximately 310 days old, that had been used previously in operant procedures. The apparatus was an operant chamber, approximately $30 \mathrm{~cm}$ on a side, fitted with a 10-cm-wide lever mounted parallel to the front wall of the chamber and $11 \mathrm{~cm}$ above the floor. Vertical lever movement of approximately $4 \mathrm{~mm}$ was sufficient to operate the lever microswitch. The lever could be fitted with easily removable counterweights to change the response effort requirement.

\section{Procedure}

All animals were given 27 days of adaptation to a feeding schedule of $1 \frac{1}{2} \mathrm{~h}$ of food every $24 \mathrm{~h}$ with ad-lib access to water. Animals were then given four daily pretraining sessions of a maximum duration of $25 \mathrm{~min}$ in which leverpresses were reinforced on a CRF schedule with one 45 -mg Noyes rat food pellet, with the lever operating force set at $10 \mathrm{~g}$. Approximately 275 reinforced presses were made by each rat during the CRF pretraining phase. The animals were then divided into two groups of 9 and 10 rats, matched on the basis of previous operant experience and on the basis of the mean time taken to complete 100 reinforced presses on the previous 2 days. One group was randomly assigned to the CRF condition and one to the FR condition. Over each of the next 6 training days, each rat made approximately 100 leverpresses

This research was partly supported by a grant from the Small Grants Program of the Graduate School of the University of Toledo. Address correspondence to Harvard L. Armus, Department of Psychology, The University of Toledo, 2801 W. Bancroft St., Toledo, OH 43606. on the appropriate reinforcement schedule, CRF or FR, with the FR rats trained up to FR-5 so that the last 4 training days were on this schedule.

On each of the next 8 days, each rat was placed into the operant chamber for $20 \mathrm{~min}$, divided into four 5-min periods, each with a different response force requirement $(10,20,35$, or $55 \mathrm{~g})$.

At the end of each 5-min period, the houselight went off, and the experimenter immediately replaced the lever counterweight with the next programmed one and reset the apparatus. This procedure typically took no longer than $3 \mathrm{sec}$.

The order of response force requirements was the same for each rat on a given day, but was varied over days so that, over the 8 testing days, each force requirement appeared in each position an equal number of times and preceded and followed every other force requirement an equal number of times.

\section{RESULTS AND DISCUSSION}

The effect of the response effort requirement was significant for both Group CRF and Group FR-5, with greater required response effort resulting in a greater ratio of responses with IRTs of less than 1 sec to total num-

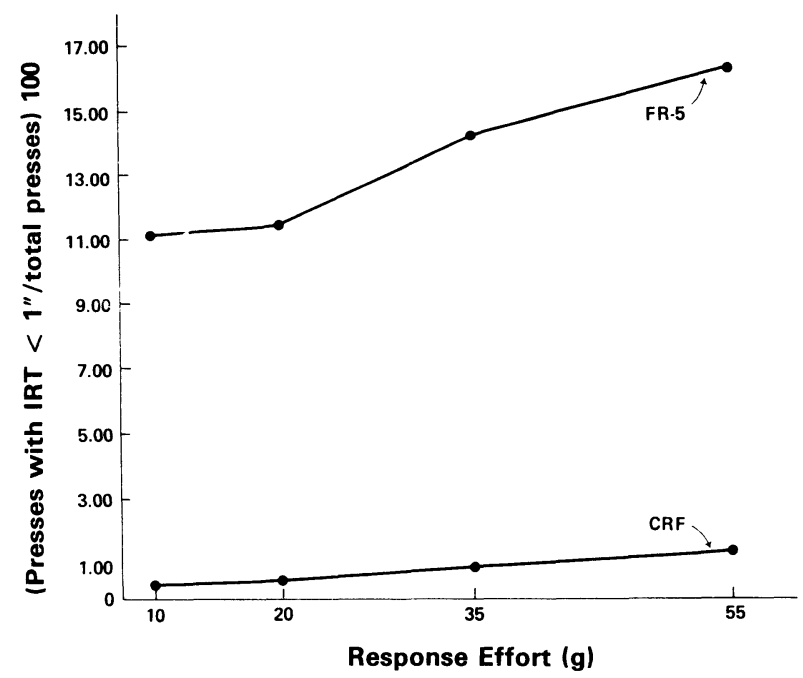

Figure 1. Ratio of presses with interresponse times of less than 1 sec to total number of presses for FR-5 and CRF groups as functions of minimum force (effort) required to activate response lever. 
ber of responses [Friedman's two-way nonparametric analysis of variance: $\chi_{r}^{2}(3)=143.48, p<.001$, and $\chi_{r}^{2}(3)$ $=167.48, p<.001$, for Group CRF and Group FR-5, respectively]. The increasing difference between the FR-5 and CRF curves with increasing required response effort was also significant $\left[\chi_{r}^{2}(3)=132.80, p<.001\right]$. These data are shown in Figure 1. In addition, Group FR-5 had a greater ratio of short to total IRTs than did Group CRF [Mann-Whitney $U$ tests, both overall and for each effort condition individually: $U(9,10)=0, p<.002]$

The results indicate that specifically training animals in multiple responses by use of a fixed-ratio schedule increased the ratio of short IRT responses to total number of responses. More important, however, was the finding that this procedure resulted in an accentuation of the ef- fect of response effort on the relative frequency of short IRT responses.

One possible explanation for the effort effect is in terms of differential salience of response-produced stimulus feedback resulting from responses requiring different degrees of effort (Armus, 1986). If it is assumed that training animals to repeat responses rapidly results in less decay of response-produced feedback cues, then a similar explanation may be proposed for the accentuated effort effect found with the FR-5 animals.

\section{REFERENCES}

Armus, H. L. (1986). Effect of response effort requirement on frequency of short interresponse times. Bulletin of the Psychonomic Society, 24, 284-285. 